\title{
Functional Polymeric Coatings for CsI(T1) Scintillators
}

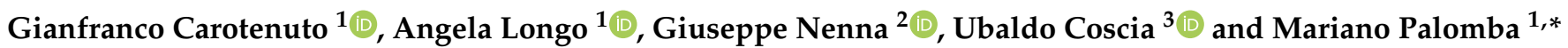 \\ 1 Institute for Polymers, Composites and Biomaterials (IPCB-CNR), National Research Council, \\ Piazzale E. Fermi 1, I-80055 Portici, NA, Italy; giancaro@unina.it (G.C.); angela.longo@cnr.it (A.L.) \\ 2 Italian National Agency for New Technologies, Energy and Sustainable Economic Development (ENEA), \\ Research Centre Portici, Piazzale E. Fermi 1, I-80055 Portici, NA, Italy; giuseppe.nenna@enea.it \\ 3 Department of Physics 'Ettore Pancini', University of Naples 'Federico II', Via Cintia, \\ I-80126 Napoli, NA, Italy; ubaldo.coscia@unina.it \\ * Correspondence: mariano.palomba@cnr.it
}

Citation: Carotenuto, G.; Longo, A.; Nenna, G.; Coscia, U.; Palomba, M. Functional Polymeric Coatings for CsI(Tl) Scintillators. Coatings 2021, 11, 1279. https://doi.org/10.3390/ coatings11111279

Academic Editor: Je Moon Yun

Received: 16 September 2021

Accepted: 18 October 2021

Published: 21 October 2021

Publisher's Note: MDPI stays neutral with regard to jurisdictional claims in published maps and institutional affiliations.

Copyright: (c) 2021 by the authors. Licensee MDPI, Basel, Switzerland. This article is an open access article distributed under the terms and conditions of the Creative Commons Attribution (CC BY) license (https:// creativecommons.org/licenses/by/ $4.0 /)$.

\begin{abstract}
The handling of inorganic scintillators (e.g., alkali metal halides) can benefit from the availability of polymeric materials able to adhere to their surface. Polymeric materials, such as epoxy resins, can act as protective coatings, as adhesives for photodiodes to be connected with the scintillator surface, and as a matrix for functional fillers to improve the optical properties of scintillators. Here, the optical properties of two epoxy resins (E-30 by Prochima, and Technovit Epox by Heraeus Kulzer) deposited on the surface of a scintillator crystal made of CsI(Tl) were investigated, in order to improve the detection of high-energy radiation. It is found that these resins are capable of adhering to the surface of alkali metal halides. Adhesion, active at the epoxy-CsI(Tl) interface, can be explained on the basis of Coulomb forces acting between the ionic solid surface and an ionic intermediate of synthesis generated during the epoxy setting reaction. Technovit Epox showed higher transparency, and it was also functionalized by embedding white powdered pigments (PTFE or $\mathrm{BaSO}_{4}$ ) to achieve an optically reflective coating on the scintillator surface.
\end{abstract}

Keywords: optical-grade epoxy; inorganic scintillator; alkali metal halides; adhesion; interface; Coulomb forces; optical properties

\section{Introduction}

Alkali metal halide crystals, such as $\mathrm{NaCl}, \mathrm{NaI}(\mathrm{Tl}), \mathrm{CsI}$, and $\mathrm{CsI}(\mathrm{Tl})$, are excellent optical windows and scintillator materials to detect high-energy radiation (e.g., $\gamma$-rays, and X-rays) [1-13]. These inorganic scintillators offer (i) a high output and energy resolution, (ii) a fast and high linear response, and (iii) a very stable light output over a wide range of temperatures; however, they are moisture sensitive and, therefore, quite difficult to handle [4]. In addition, the difficult processing of these materials strongly limits their technological exploitation. Usually, to solve these problems, the crystal is covered by PTFE tape; however, this technological approach has several limitations (tape breaking, non-uniform shape, etc.). Therefore, in this paper, we propose replacing the tape with structural adhesive polymeric materials such as epoxy resins containing reflective powders.

It is known that ionic scintillators are solids made of close-packed alkali metal cations and halogen anions, interacting by Coulomb's electrostatic forces, and adhesion is not a critical issue for ionic solids such as ceramic materials (e.g., potteries and glasses) since their surfaces have a layer of hydroxyl groups that allows a strong grafting with the adhesive phase [14]. However, such a layer is not present on the surface of an alkali metal halide crystal, and therefore the polymer-crystal interfacial adhesion is a very critical issue for these materials.

It is well known that polymers have a more or less effective capability of adhering to different solid surfaces by physical or even chemical interactions, depending on the type of side group [15]. For such a reason, the adhesion of thermoplastic polymers to an ionic surface should improve upon increasing the side group polarity, but a mechanically 
stable polymer-ionic solid interface can never be achieved by ion-dipole interactions. On the other hand, epoxy resin is an almost universal structural adhesive class, able to guarantee a strong interface with solids such as ceramics, inorganic glasses, metals, and even most plastics [16,17]. Since ionic groups (alkoxide and ammonium) are generated in the epoxy structure during the setting reaction [18], these materials can determine an effective interaction with the cations/anions present on the alkali metal halide surface, which provides the adhesion with the crystal.

Here, the adhesion of two optical-grade epoxy resins to a CsI(Tl) crystal was tested after their characterization by UV-Vis spectroscopy, fluorescence spectroscopy, scanning electron microscopy (SEM), and energy-dispersive X-ray spectroscopy (EDS). To improve the reflectance of the coating layer, these resins were filled by white organic/inorganic pigments of PTFE or $\mathrm{BaSO}_{4}$.

\section{Materials and Methods}

The experimental activity was focused on the surface modification of inorganic scintillators, based on thallium-doped cesium iodide, CsI(Tl), monocrystals, by epoxy resins. The aim of the surface treatment was the protection/functionalization of the scintillator surface, and it was carried out on single crystals, having a cubic shape with a side of $35 \mathrm{~mm}$. Since CsI is highly hygroscopic and sensitive to oxidation, the scintillators were stored in a desiccator with well-activated silica gel. Two types of optical-grade epoxies: E-30 by Prochima and Technovit Epox by Heraeus Kulzer, were tested as scintillator adhesives. The base resin-to-hardener weight ratio was 5:3 for the E-30 epoxy resin, and 3:1 for Technovit Epox. In the case of Technovit Epox, a fast hardener was used. An as-prepared epoxy resin mixture was applied by spraying it on the crystal surface. After that, to eliminate air bubbles and other defects formed in the deposited layer, the covered crystals were kept in oven, under vacuum, at a temperature of $40^{\circ} \mathrm{C}$ (for E-30) and $80^{\circ} \mathrm{C}$ (for Technovit Epox), for ca. $2 \mathrm{~h}$, and then the samples were left to cure in air for 2 days. Teflon nanopowder (PTFE, Aldrich, St. Louis, MO, USA) and barium sulphate $\left(\mathrm{BaSO}_{4}, 99 \%\right.$, Alfa-Aesar, Haverhill, MA, USA) were selected as a white reflective filler for Technovit Epox. In particular, the filler suspension in the base resin component was treated by an ultrasonic bath for $30 \mathrm{~min}$ to improve dispersion. After the hardener addition to the base epoxy, accurate mixture stirring, and its deposition on the CsI(Tl) crystal, the coating was allowed to cure at a temperature of $80^{\circ} \mathrm{C}$ for $2 \mathrm{~h}$. To obtain an optimal compromise between visible reflectivity and coating uniformity, it was required for the epoxy-based composites were required to be filled with $5.4 \%$ by weight of PTFE or $21.3 \%$ by weight of $\mathrm{BaSO}_{4}$.

Microscopic characterization and elemental analysis were performed by a scanning electron microscope (FEI Quanta 200 FEG microscope, Theromo Fischer Scientific, Hillsboro, OR, USA )and energy-dispersive X-ray spectroscopy (Inca Oxford 250, Oxford, UK), respectively. Absorption and emission optical spectroscopy measurements were conducted by a UV-Vis-NIR spectrophotometer (PerkinElmer, Lambda-900, Hong Kong, China), equipped with an integrating sphere (diameter of $15 \mathrm{~cm}$, PerkinElmer, Hong Kong, China), covered by Spectralon, and a spectrofluorometer (PerkinElmer, LS-55, Hong Kong, China), respectively.

\section{Results and Discussion}

The surface morphology of the "as-received" CsI(Tl) crystals was investigated by scanning electron microscopy (SEM, FEI Quanta 200 FEG microscope, Theromo Fischer Scientific, Hillsboro, OR, USA), and energy-dispersive X-ray spectroscopy (EDS) was used in order to quantify the surface oxidation extent. Figure 1a shows the SEM micrograph of the fractured crystal surface. In the inset, the brittle fracture clearly evidences the many dislocations present in the crystal lattice. The EDS spectrum of the fractured surface is shown in Figure $1 \mathrm{~b}$ where intensive signals of cesium and iodine elements can be appreciated, while the thallium signal is not visible because it is present in a very small 
amount. A low-intensity oxygen signal is also present, indicating a slight surface oxidation, and the very small carbon peak is due to the SEM sample preparation.
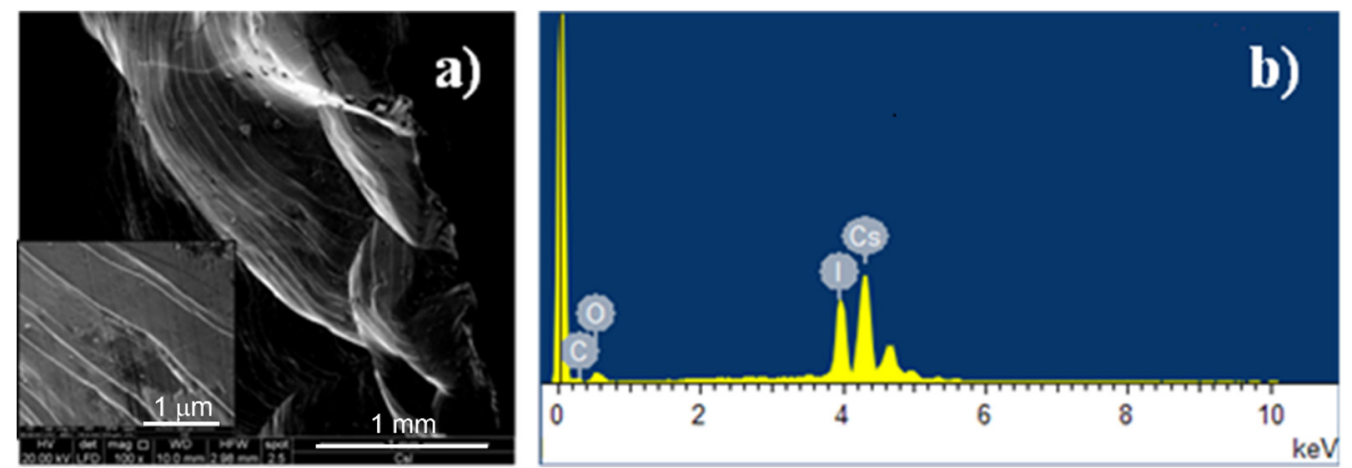

Figure 1. SEM micrograph of the CsI(Tl) crystal surface (a) and related EDS spectrum (b). The inset of (a) shows a magnification of the surface dislocations.

The absorption properties of the CsI(Tl) crystal were measured by absorption spectroscopy. As shown in Figure 2a, even a very thin slice of CsI(Tl) showed a rapidly saturating absorption band starting at $315 \mathrm{~nm}$, while the sample was uniformly transparent in the 320-800 nm spectral range.
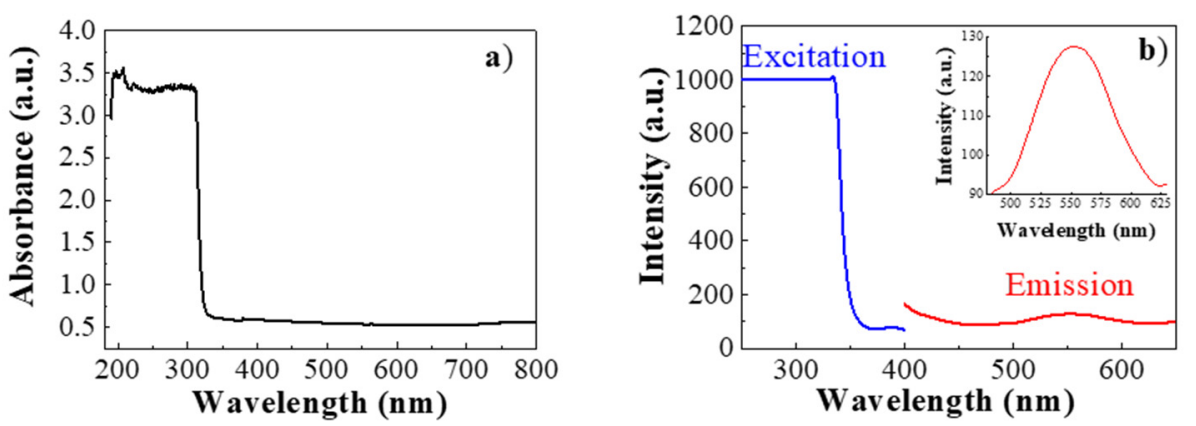

Figure 2. UV-Vis absorption spectrum of $\mathrm{CsI}(\mathrm{Tl})$ (slice of $0.883 \mathrm{~mm}$ ) (a), and UV-Vis excitationemission spectra of $\mathrm{CsI}(\mathrm{Tl})$ (b). In the inset, a magnification of the emission peak is shown.

The fluorescence characteristics of the scintillator crystals were explored by fluorimetry. As shown in Figure 2b, one visible light emission of green color was found under ultraviolet excitation. In particular, the excitation spectrum (blue curve) shows a cut-off in the UV region, while the emission spectrum (red curve) presents a symmetric peak, centered at $550 \mathrm{~nm}$ (see the inset of Figure 2b).

For optimal scintillator operation, it is required to avoid the full attenuation of the fluorescence signal emitted under exposure to high-energy radiation ( $\gamma$-rays, X-rays, etc.). Therefore, the optical transparency of the epoxy resins (E-30 by Prochima and Technovit Epox by Heraeus Kulzer) in the green spectral range (520-560 nm) was measured. As it can be seen in Figure 3, after curing, these two resins, which were $0.5 \mathrm{~mm}$ thick, they had a cut-off value of $272 \mathrm{~nm}$ and $290 \mathrm{~nm}$, respectively. Both cut-off values are quite close to the CsI(Tl) crystal self-absorption wavelength, which is $315 \mathrm{~nm}$; however, the optimal optical features were found for the Technovit Epox resin because of the higher transmittance in the UV-Vis-NIR spectral range. 


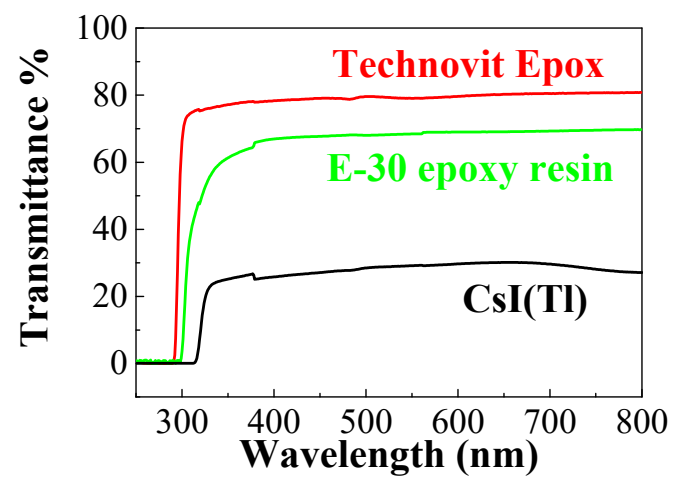

Figure 3. Transmittance spectra of the cured E-30 and Technovit Epox resins, and CsI(Tl) crystal.

Spray technology was used to deposit the Technovit Epox resin on the CsI(Tl) crystal surface. As shown in Figure 4a, a continuous and defect-free coating layer of $0.5 \mathrm{~mm}$ thickness was deposited, leading to a coated crystal with a size of $35 \mathrm{~mm}$. Such a type of coating can only slightly attenuate the fluorescent green light emitted by the scintillator crystal under UV radiation (see Figure $4 \mathrm{~b}$ ). According to the difficult peel-out of the Technovit Epox coating, a mechanically robust epoxy-crystal interface resulted. This very good adhesion property could be attributed to some special chemical interaction active at the epoxy-CsI(Tl) interface.
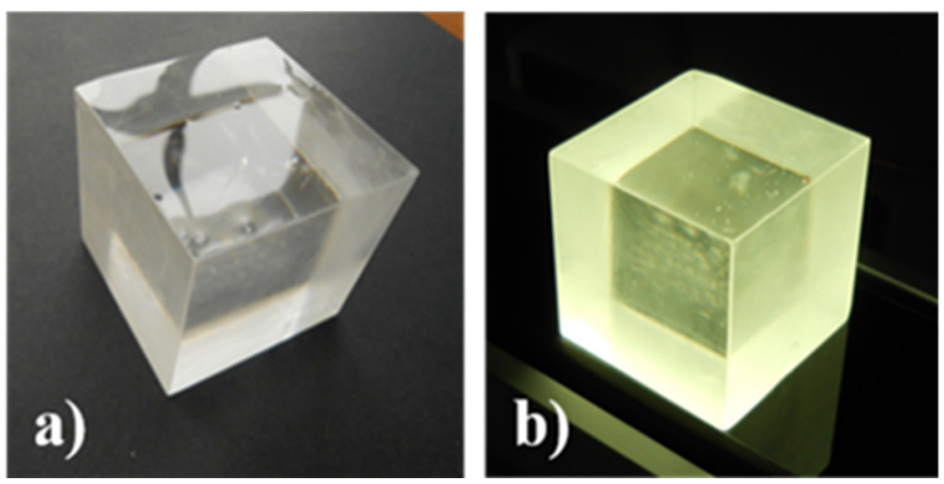

Figure 4. $\mathrm{CsI}(\mathrm{Tl})$ scintillator crystal coated by Technovit Epox resin under visible (a) and UV light (b).

In this work, it was observed that epoxy resin showed good adhesion to the CsI(Tl) crystal surface even without preliminary treatments. Such bonding can be explained on the basis of the formation, during the curing reaction, of an ionic intermediate of synthesis (i.e., $-\mathrm{CH}\left(\mathrm{O}^{-}\right)-\mathrm{CH}_{2}-\mathrm{NH}_{2}{ }^{+}$) that could persist at the organic/inorganic interface because of electrostatic interactions with the $\mathrm{Cs}^{+}$and $\mathrm{I}^{-}$ions present on the crystal surface.

To improve the collection of the emitted fluorescence signal by multiple light reflections, the possibility of filling the epoxy layer with a white pigment was also verified. The deposition of such a functionalized coating layer increases the reflectance of the crystal walls.

In the case of the white pigment based on PTFE nanopowder, a not very uniform dispersion was obtained for a filling factor higher than $5.4 \%$ by weight, Figure 5 a, and large aggregates of PTFE grains appeared in the deposited layer, Figure 5b. 

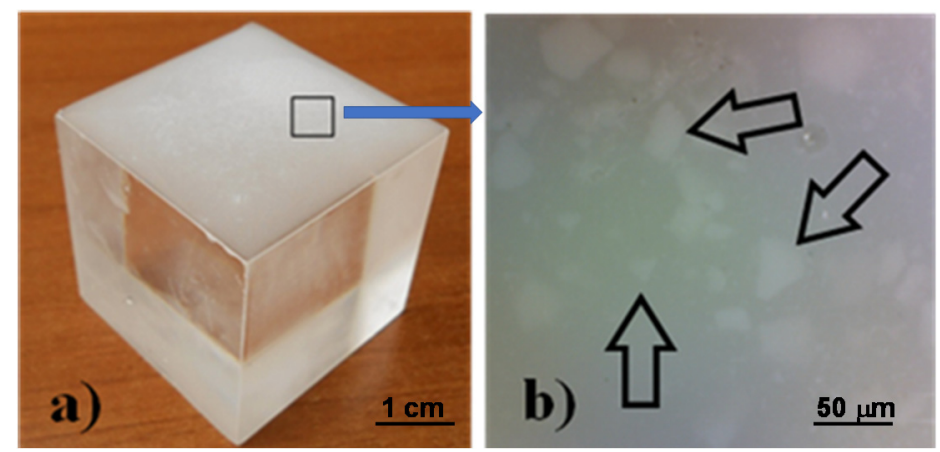

Figure 5. Surface of a CsI(Tl) scintillator coated by a reflective layer of Technovit Epox resin filled with PTFE nanopowder (a). An optical micrograph of the achieved surface microstructure, where the arrows indicate the aggregates present in the coating layer $(\mathbf{b})$.

To improve the coating uniformity, $\mathrm{BaSO}_{4}$ powder was tested as a filler, since it is a white reflective pigment widely used in optics [19-21]. According to the SEM micrograph and the EDS spectrum shown in Figure $6 \mathrm{a}, \mathrm{b}$, the $\mathrm{BaSO}_{4}$ powder had an average size of $431 \mathrm{~nm}$ and contained copper impurity ( $2.3 \%$ by weight).
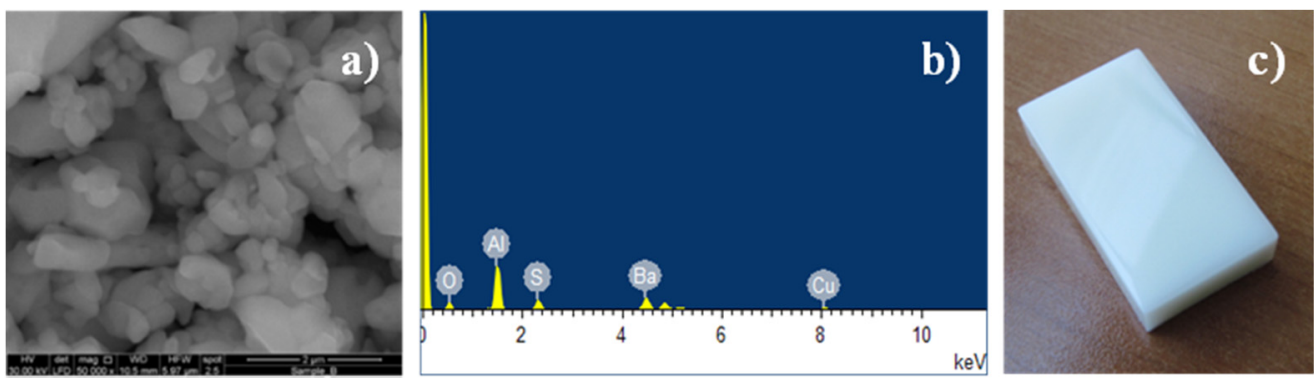

Figure 6. SEM micrograph of the $\mathrm{BaSO}_{4}$ powder (a), related EDS spectrum (b), and $\mathrm{BaSO}_{4}$ / epoxy sample image (c).

When sonication was applied during the preparation of the epoxy $/ \mathrm{BaSO}_{4}$ mixture, a much higher filling factor was achieved ( $21.3 \%$ by weight), without observing significant grain aggregation in the coating (see Figure 6c).

A reasonably good result was achieved by filling the epoxy resin with $\mathrm{BaSO}_{4}$ powder. Figure 7 shows the total reflectance spectrum in the visible range of the pure $\mathrm{CsI}(\mathrm{Tl})$ scintillator crystal (blue curve) and the $\mathrm{CsI}(\mathrm{Tl})$ scintillator crystal coated by a $\mathrm{BaSO}_{4} /$ epoxy layer (green curve). These optical measurements clearly show a higher reflectance value for the coated crystal.

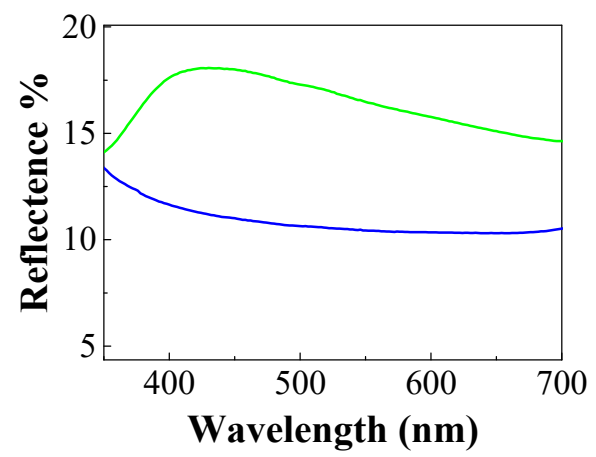

Figure 7. Total reflectance spectra of: CsI(Tl) scintillator (blue curve) and CsI(Tl) scintillator coated by a $\mathrm{BaSO}_{4}$ /epoxy layer (green curve). 


\section{Conclusions}

The optical characteristics of two commercial optical-grade epoxy resins used as coatings for $\mathrm{CsI}(\mathrm{Tl})$ crystals were compared. The best optical properties were found for Technovit Epox that also showed good adhesion characteristics for the CsI(Tl) crystal surface. Such adhesive properties of epoxies toward alkali metal halides could be ascribed to the possibility for these macromolecules to generate, during the setting reaction, an ionic intermediate that may electrostatically interact with cations and anions at the salt-resin interface. The functionalization the epoxy coating with a white pigment $\left(\mathrm{BaSO}_{4}\right.$ powder), in order to improve the reflectance on the scintillator surface in the visible spectral region, was also investigated.

Author Contributions: Conceptualization, G.C.; methodology, G.C.; validation, G.C. and U.C.; formal analysis, A.L., M.P. and G.N.; investigation, G.C., A.L., M.P. and G.N.; data curation, G.C., A.L. and M.P.; writing — original draft preparation, G.C., A.L. and M.P.; writing—review and editing, G.C., A.L., M.P., U.C. and G.N. All authors have read and agreed to the published version of the manuscript.

Funding: This research received no external funding.

Institutional Review Board Statement: Not applicable.

Informed Consent Statement: Not applicable.

Data Availability Statement: Not applicable.

Acknowledgments: The authors kindly acknowledge the projects titled: "Nanocompositi polimerici per applicazione ottiche" (CNR) and "Calocube" (INFN). The authors are grateful to Maria Cristina Del Barone of the LAMEST laboratory (IPCB-CNR) for the SEM/EDS measurements.

Conflicts of Interest: The authors declare no conflict of interest.

\section{References}

1. Baldochi, S.L.; Ranieri, I.M. Encyclopedia of Materials: Science and Technology; Elsevier: Amsterdam, The Netherlands, 2001; pp. 74-78.

2. Phillips, W.M.; Stearns, J.W. Alkali metal/halide thermal energy storage systems performance evaluation. J. Sol. Energy Eng. 1987, 109, 235-237. [CrossRef]

3. Bourret-Courchesne, E.D.; Bizarri, G.A.; Borade, R.; Gundiah, G.; Samulon, E.C.; Yan, Z.; Derenzo, S.E. Crystal growth and characterization of alkali-earth halide scintillators. J. Cryst. Growth 2012, 352, 78-83. [CrossRef]

4. Yanagida, T. Inorganic scintillating materials and scintillation detectors. Proc. Jpn. Acad. Ser. B 2018, 94, 75-97. [CrossRef] [PubMed]

5. Kubota, S.; Sakuragi, S.; Hashimoto, S.; Ruan, J.-Z. A new scintillation material: Pure CsI with 10 ns decay time. Nucl. Instrum. Meth. Phys. Res. A 1988, 268, 275-277. [CrossRef]

6. Adriani, O.; Albergo, S.; Auditore, L.; Zampa, G.; Basti, A.; Berti, E.; Bigongiari, G.; Bonechi, L.; Bongi, M.; Bonvicini, V.; et al. The CALOCUBE project for a space based cosmic ray experiment: Design, construction, and first performance of a high granularity calorimeter prototype. J. Instrum. 2019, 14, P11004. [CrossRef]

7. Pacini, L.; Adriani, O.; Agnesi, A.; Zampa, G.; Zampa, N.; Auditore, L.; Basti, A.; Berti, E.; Bigongiari, G.; Bonechi, L.; et al. CaloCube: An innovative homogeneous calorimeter for the next-generation space experiments. J. Phys. Conf. Ser. 2017, 928, 012013. [CrossRef]

8. Kurman, Y.; Shultzman, A.; Segal, O.; Pick, A.; Kaminer, I. Photonic-crystal scintillators: Molding the flow of light to enhance $\mathrm{X}$-ray and $\gamma$-ray detection. Phys. Rev. Lett. 2020, 125, 040801. [CrossRef] [PubMed]

9. Cabanelas, P.; González, D.; Alvarez-Pol, H.; Boillos, J.M.; Casarejos, E.; Cederkall, J.; Cortina, D.; Feijoo, M.; Galaviz, D.; Galiana, E.; et al. Performance recovery of long CsI(Tl) scintillator crystals with APD-based readout. Nucl. Instrum. Methods Phys. Res. A 2020, 965, 163845. [CrossRef]

10. Gramuglia, F.; Frasca, S.; Ripiccini, E.; Venialgo, E.; Gâté, V.; Kadiri, H.; Descharmes, N.; Turover, D.; Charbon, E.; Bruschini, C.; et al. Light extraction enhancement techniques for inorganic scintillators. Crystals 2021, 11, 362. [CrossRef]

11. Li, G.; Lou, J.L.; Ye, Y.L.; Hua, H.; Wang, H.; Han, J.X.; Huang, M.R. Property investigation of the wedge-shaped CsI (Tl) crystals for a charged-particle telescope. Nucl. Instrum. Methods Phys. Res. A 2021, 1013, 165637. [CrossRef]

12. Knyazev, A.; Park, J.; Golubev, P.; Cederkäll, J.; Alvarez-Pol, H.; Benlliure, J.; Timm, R. Simulations of light collection in long tapered CsI (Tl) scintillators using real crystal surface data and comparisons to measurement. Nucl. Instrum. Methods Phys. Res. Sect. A 2021, 1003, 165302. [CrossRef] 
13. Chen, M.; Sun, L.; Ou, X.; Yang, H.; Liu, X.; Dong, H.; Duan, X. Organic semiconductor single crystals for X-ray imaging. Adv. Mater. 2021, 2104749. [CrossRef] [PubMed]

14. Centelles, X.; Castro, J.R.; Cabeza, L.F. Experimental results of mechanical, adhesive, and laminated connections for laminated glass elements-A review. Eng. Struct. 2019, 180, 192-204. [CrossRef]

15. Wypych, G. Handbook of Adhesion Promoters; ChemTec Publishing: Scarborough, ON, Canada, 2018; Chapter 2; pp. 5-44.

16. Jin, F.-L.; Li, X. Synthesis and application of epoxy resins: A review. J. Ind. Eng. Chem. 2015, 29, 1-11. [CrossRef]

17. May, C. Epoxy Resins: Chemistry and Technology; Routledge: Oxfordshire, UK, 2018.

18. Li, X.S.; Li, M.-S.; Chang, F.-C. Kinetics and curing mechanism of epoxy and boron trifluoride monoethyl amine complex system. J. Polym. Sci. Part A 1999, 37, 3614-3624. [CrossRef]

19. Mikhailov, M.M.; Yuryev, S.A.; Lapin, A.N.; Lovitskiy, A.A. The effects of heating on $\mathrm{BaSO}_{4}$ powders' diffuse reflectance spectra and radiation stability. Dye. Pigment. 2019, 163, 420-424. [CrossRef]

20. Mikhailov, M.M.; Yuryev, S.A.; Lapin, A.N. Modelling degradation of optical properties for $\mathrm{BaSO}_{4}$ pigments modified by nanoparticles under irradiation with charged particles. Nucl. Instrum. Methods Phys. Res. Sect. B 2019, 458, 33-38. [CrossRef]

21. Li, X.; Peoples, J.; Yao, P.; Ruan, X. Ultrawhite $\mathrm{BaSO}_{4}$ paints and films for remarkable daytime subambient radiative cooling. ACS Appl. Mater. Interfaces 2021, 13, 21733-21739. [CrossRef] [PubMed] 